\title{
FINITE-TIME BLOW-UP IN THE ADDITIVE SUPERCRITICAL STOCHASTIC NONLINEAR SCHRÖDINGER EQUATION : THE REAL NOISE CASE
}

\author{
A. DE BOUARD AND A. DEBUSSCHE
}

\begin{abstract}
We review some results concerning the apparition of finite time singularities in nonlinear Schrödinger equations with a Gaussian additive noise which is white in time and correlated in space. We then extend the results to the case where the noise is real valued, which is the case in some physical situations.
\end{abstract}

\section{INTRODUCTION}

The nonlinear Shrödinger (NLS) equation is a generic equation describing the propagation of weakly nonlinear waves in strongly dispersive media. It is found in diverse fields of physics, such as hydrodynamics, plasma physics, nonlinear optics, or molecular biology, where it appears to be the continuum limit of certain discrete systems (see [2] and the references therein).

Recently, interest has grown up in the influence of Gaussian white noise on the dynamical behaviour of solutions of this equation; especially, in the focusing case, propagation of soliton solutions in the presence of noise has been the subject of several investigations.

A one dimensional NLS equation with additive Gaussian space-time white noise is e.g. considered in [7], with the aim of computing error probability in signal transmissions.

Another example of NLS equation with noise is given in [1] and [2], where it describes energy transfer in monolayer molecular aggregates, and where the noise stands for thermal fluctuations. As explained in [2], this noise may be multiplicative if it describes process where excitation is not being created or destroyed and in this case the noise appears in the equation as a linear potential. It may also be additive in the case of and exciton that creates or absorbs a photon. In both cases, the noise is real valued and depends on space and time variables.

Here, we consider the stochastic nonlinear Schrödinger equation

$$
i \partial_{t} \psi-\left(\Delta \psi+|\psi|^{2 \sigma} \psi\right)=\xi
$$

in general dimension $n$. The noise $\xi$ is an additive real valued Gaussian noise, which is white in time and correlated in space. The nonlinear term $|\psi|^{2 \sigma} \psi$ is a supercritical power of the solution, and our aim is to investigate the possible blow-up of solutions.

It is well known indeed that when there is no noise, i.e. $\xi=0$ in equation (1.1), and when $\sigma$ satisfies $\frac{2}{n} \leq \sigma<\frac{2}{n-2}\left(\sigma \geq \frac{2}{n}\right.$ in dimension $n=1$ or 2$)$, a solution of

1991 Mathematics Subject Classification. 35Q55, 60H15, 76B35.

Key words and phrases. Nonlinear Schrödinger Equations, Stochastic Partial Differential Equations, White Noise, Blow up, Variance Identity. 
(1.1) (with $\xi=0$ ) starting from $\psi_{0}$ with a finite negative energy, that is with

$$
H\left(\psi_{0}\right)=\frac{1}{2} \int\left|\nabla \psi_{0}(x)\right|^{2} d x-\frac{1}{2 \sigma+2} \int\left|\psi_{0}(x)\right|^{2 \sigma+2} d x<0
$$

cannot be globally well defined. More precisely, there is a positive $t^{*}$ such that

$$
\lim _{t \nearrow t^{*}} \int_{\mathbb{R}^{n}}|\nabla \psi(t, x)|^{2} d x=+\infty .
$$

The ingredient of the proof of such a fact uses what is sometimes called the "variance identity" (see [8], [11], [12]) which consists in computing the second order time derivative of the quantity

$$
V(\psi(t))=\int_{\mathbb{R}^{n}}|x|^{2}|\psi(t, x)|^{2} d x .
$$

Using the equation satisfied by $\psi$ and the fact that the energy $H$ is a conserved quantity for the deterministic equation, it is indeed possible to show that under the preceding conditions on $\sigma$,

$$
V(\psi(t)) \leq V\left(\psi_{0}\right)+\left.t \frac{d}{d t} V(\psi(t))\right|_{t=t_{0}}+8 H\left(\psi_{0}\right) t^{2}
$$

$V(\psi)$ being a nonegative quantity, this inequality cannot remain true for all time if e.g. the energy $H\left(\psi_{0}\right)$ is negative, and it leads to (1.2). The condition $H\left(\psi_{0}\right)<0$ is of course far from necessary in order that the solution blows up, and some much more precise criteria may be exhibited (see [10]).

We have generalized in [5] this identity to the stochastic equation (1.1), where $\xi$ is a complex valued noise which is correlated in space and white in time. In this case, the solution is a random process, which is defined on a random time interval $\left[0, \tau^{*}\left(\psi_{0}\right)\right)$, provided that $\psi$ is sufficiently correlated in space, as was proved in [4]. Assuming that for some deterministic $t>0$, one has $t<\tau^{*}\left(\psi_{0}\right)$ almost surely and that

$$
\mathbb{E} \int_{0}^{t}\left(\int_{\mathbb{R}^{n}}|\nabla \psi(s, x)|^{2} d x+\left(\int_{\mathbb{R}^{n}}|\psi(s, x)|^{2 \sigma+2} d x\right)^{\frac{2 \sigma+1}{\sigma+1}} d s\right)<+\infty
$$

we have proved that $\mathbb{E}(V(\psi(t)))$ satisfies an inequality of the form (1.3) where the right hand side is replaced by a third order polynomial in time; here, the expression $\mathbb{E}(v)$ stands for the mathematical expectation, or mean value, of the random variable $v$. In this third order polynomial, the coefficient of $t^{3}$ depends only on the covariance operator of the noise, while the coefficient of $t^{2}$ is $8 \mathbb{E}\left(H\left(\psi_{0}\right)\right)$. Hence, by choosing $\psi_{0}$ such that $\mathbb{E}\left(H\left(\psi_{0}\right)\right)$ is sufficiently negative, again the inequality cannot remain true for all positive time because the right hand side takes negative values, and the solution necessarily blows up (see Proposition 2.3 for a precise definition of blow-up).

This result does not make use of the fact that the noise is complex valued, and it is true with exactly the same proof in the present case of a real valued noise.

We then made use in [5] of a control argument to show that if the noise is nondegenerate, the stochastic equation is irreducible in the sense that for any time $T>0$, initial data $\psi_{0}$ and final data $\psi_{T}$, the solution of (1.1) with $\psi(0)=\psi_{0}$ is close at time $T$ to $\psi_{T}$ with a positive probability. Choosing then $\psi_{T}$ with sufficiently negative energy allowed us to apply the stochastic variance identity, to the solution 
of (1.1) starting from $\psi_{T}$ at time $T$, so that this solution finally blows up. Hence, blow-up occurs for any initial data.

Contrary to the variance identity, the control argument leading to the irreducibility of the equation strongly uses the fact that the noise is complex valued, since in this case it is sufficient to control the equation with a complex valued deterministic forcing term. The aim of the present note is first to review the existence and blow-up results that were previously obtained and then to show that the control argument is still valid in the real valued case, implying the same result as in the complex valued case, that is any solution of (1.1) blows up in finite time if the noise is sufficiently correlated.

Note that on the opposite case of a completely uncorrelated noise in space - that is a space-time white noise - even though we are not able to prove any theoretical result, some numerical computations have been performed in [6], which seem to indicate that if the noise is multiplicative and arises as a Stratonovitch potential then it will tend to prevent the blow-up phenomenon.

We now describe more precisely the noise that we consider. We introduce a probability space $(\Omega, \mathcal{F}, \mathbb{P})$, endowed with a filtration $\left(\mathcal{F}_{t}\right)_{t \geq 0}$, and a sequence $\left(\beta_{k}\right)_{k \in \mathbb{N}}$ of independent real valued Brownian motions on $\mathbb{R}^{+}$associated to the filtration $\left(\mathcal{F}_{t}\right)_{t \geq 0}$. We then consider a complete orthonormal system $\left(e_{k}\right)_{k \in \mathbb{N}}$ in the space of real valued square integrable functions on $\mathbb{R}^{n}$, and a bounded linear operator $\Phi$ on this space. The process

$$
W(t, x, \omega)=\sum_{k=0}^{\infty} \beta_{k}(t, \omega) \Phi e_{k}(x), \quad t \geq 0, \quad x \in \mathbb{R}^{n}, \quad \omega \in \Omega,
$$

is then a Wiener process on the space of real valued square integrable functions on $\mathbb{R}^{n}$, with covariance operator $t \Phi \Phi^{*}$. We then set $\xi=\frac{\partial W}{\partial t}$. Note that if $\Phi$ is defined through a real valued kernel $\mathcal{K}$, which means that for any real valued square integrable function $u$,

$$
\Phi u(x)=\int_{\mathbb{R}^{n}} \mathcal{K}(x, y) u(y) d y,
$$

then the correlation function of the noise is given by

$$
\mathbb{E}\left(\frac{\partial W}{\partial t}(t, x) \frac{\partial W}{\partial t}(s, y)\right)=c(x, y) \delta_{t-s}
$$

with

$$
c(x, y)=\int_{\mathbb{R}^{n}} \mathcal{K}(x, z) \mathcal{K}(y, z) d z .
$$

We then write equation (1.1) as

$$
i d \psi-\left(\Delta \psi+|\psi|^{2 \sigma} \psi\right) d t=d W .
$$

Note that in the physical situations described at the beginning, the correlation function $c(x, y)$ is a Dirac delta function, corresponding to space-time white noise (in this case, $\Phi$ is the identical operator). We are not able to treat that case for two reasons. To understand them, one should consider the linear equation

$$
\left\{\begin{array}{l}
i d z-\Delta z d t=d W \\
z(0)=0
\end{array}\right.
$$


whose solution is given by the stochastic integral

$$
z(t)=\int_{0}^{t} S(t-s) d W(s)
$$

where $S(t)=e^{-i t \Delta}$ is the group associated with the linear Schrödinger equation. Since $S(t)$ is a unitary group in any Sobolev space $H^{s}\left(\mathbb{R}^{n}\right)$, it is easy to see that $z(t)$ lies in $H^{s}\left(\mathbb{R}^{n}\right)$ almost surely if and only if $\Phi$ is a Hilbert-Schmidt operator from $L^{2}\left(\mathbb{R}^{n}\right)$ into $H^{s}\left(\mathbb{R}^{n}\right)$. Note indeed the identity

$$
|z(t)|_{H^{s}\left(\mathbb{R}^{n}\right)}^{2}=\left|\int_{0}^{t} S(t-s) d W(s)\right|_{H^{s}\left(\mathbb{R}^{n}\right)}^{2}=t\|\Phi\|_{H S\left(L^{2}, H^{s}\right)}^{2}
$$

where $\|\Phi\|_{H S\left(L^{2}, H^{s}\right)}^{2} \equiv \sum_{k}\left|\Phi e_{k}\right|_{H^{s}\left(\mathbb{R}^{n}\right)}^{2}$ is the Hilbert-Schmidt norm of $\Phi$ as an operator from $L^{2}\left(\mathbb{R}^{n}\right)$ into $H^{s}\left(\mathbb{R}^{n}\right)$.

However, it is easy to see that a convolution operator - i.e. an operator defined through a kernel $\mathcal{K}(x, y)=k(x-y)$ - will never be Hilbert-Schmidt from $L^{2}\left(\mathbb{R}^{n}\right)$ into $H^{s}\left(\mathbb{R}^{n}\right)$, even if $s$ is largely negative. This proves that the integral $z(t)$ cannot live in $H^{s}\left(\mathbb{R}^{n}\right)$ if the noise is homogeneous. This is the first reason : homogeneity of the noise.

The second reason is the irregularity of the correlations : even if one adds some localization in the correlations of the noise - e.g. if $\Phi$ is given by a kernel $\mathcal{K}(x, y)=k(x) \delta_{x-y}$ - there is no hope that $z(t)$ lies in a more regular space than $H^{-n / 2}\left(\mathbb{R}^{n}\right)$. However, it has been proved (see [9]) that the deterministic conservative NLS equation is ill posed in any $H^{s}\left(\mathbb{R}^{n}\right)$ with negative $s$.

This implies in particular that treating the stochastic term as a perturbation by using the integral $z(t)$ will never lead to the existence of a strong solution of the stochastic equation with a space-time white noise, as long as we deal with $H^{s}$ Sobolev spaces ; note that the $H^{s}$ spaces have revealed to be very natural spaces to handle the deterministic NLS equation.

Anyway, we only consider correlated noise in this note, which means that we will require from $\Phi$ sufficient regularization properties, and the above mentionned problem will not appear here.

The note is organized as follows : in Section 2, we recall the results proved in [4] and [5] concerning the existence of solutions and blow-up for some initial data. Those results were proved in the context of a complex valued noise, but they hold with exactly the same proof for equation (1.4) with a real valued noise, so that we do not recall the proofs. we will give for each particular result the minimal assumptions required on $\Phi$, and on the initial data. In Section 3, we prove that the controlability problem allowing to deduce the irreducibility of equation (1.4) has a solution in the real valued case - and here the proof is different and more complicated than in the complex valued case. We then deduce the irreducibility as in [5] from this result and from the continuity with respect to the forcing term in the equation. Finally, irreducibility together with the blow-up result of Section 2 implies as in [5] the blow-up for any initial data (see Theorem 3.1).

2. REVIEW OF EXISTENCE AND BLOW-UP FOR A RESTRICTED CLASS OF INITIAL DATA

We start with some local and global existence results. All these results are proved in [4]. 


\subsection{Local and global existence results.}

Theorem 2.1. Assume that $0 \leq \sigma<2 /(n-2)$ if $n \geq 3$ or $0 \leq \sigma$ for $n=1,2$, that $\Phi$ is Hilbert-Schmidt from $L^{2}\left(\mathbb{R}^{n}\right)$ into $H^{1}\left(\mathbb{R}^{n}\right)$ and that the initial data $\psi_{0}$ is a $\mathcal{F}_{0}$ measurable random variable with values in $H^{1}\left(\mathbb{R}^{n}\right)$; then there exists a unique solution $\psi$ to (1.4) with continuous $H^{1}$ valued paths, such that $\psi(0)=\psi_{0}$. This solution is defined on a random interval $\left[0, \tau^{*}\left(\psi_{0}\right)\right)$, where $\tau^{*}\left(\psi_{0}\right)$ is a stopping time such that

$$
\tau^{*}\left(\psi_{0}\right)=+\infty \text { or } \lim _{t / \tau^{*}\left(\psi_{0}\right)}|\psi(t)|_{H^{1}\left(\mathbb{R}^{n}\right)}=+\infty .
$$

Furthermore, $\tau^{*}$ is almost surely lower semicontinuous with respect to $\psi_{0}$.

In order to prove the global existence result in the subcritical case $\sigma<2 / n$, the following invariant quantities of the deterministic NLS equation have been used in [4] : the momentum

and the Hamiltonian

$$
M(\psi)=\int_{\mathbb{R}^{n}}|\psi(x)|^{2} d x
$$

$$
H(\psi)=\frac{1}{2} \int_{\mathbb{R}^{n}}|\nabla \psi(x)|^{2} d x-\frac{1}{2 \sigma+2} \int_{\mathbb{R}^{n}}|\psi(x)|^{2 \sigma+2} d x .
$$

The evolution of these quantities along the solutions of the stochastic equation (1.4) is described in the next proposition.

Proposition 2.1. Let $\psi_{0}, \sigma$ and $\Phi$ be as in Theorem 2.1. For any stopping time $\tau$ such that $\tau<\tau^{*}\left(\psi_{0}\right)$ a.s., we have

$$
M(\psi(\tau))=M\left(\psi_{0}\right)-2 \operatorname{Im} \sum_{\ell \in \mathbb{N}} \int_{0}^{\tau} \int_{\mathbb{R}^{n}} \psi(x) \Phi e_{\ell}(x) d x d \beta_{\ell}(s)+\tau \sum_{\ell}\left|\Phi e_{\ell}\right|_{L^{2}}^{2}
$$

where $\psi$ is the solution of (1.4) given by Theorem 2.1 with $\psi(0)=\psi_{0}$.

Moreover, for any $k \in \mathbb{N}$,

$$
\mathbb{E}\left[\sup _{t \in[0, \tau]} M^{k}(\psi(\tau))\right] \leq C_{k} \mathbb{E}\left[M^{k}\left(\psi_{0}\right)\right]
$$

for a constant $C_{k} \geq 0$.

In the same way, for any $\tau$ such that $\tau<\tau^{*}\left(\psi_{0}\right)$ a.s. we have

$$
\begin{aligned}
H(\psi(\tau)) & =H\left(\psi_{0}\right)-\operatorname{Im} \int_{\mathbb{R}^{n}} \int_{0}^{\tau}\left(\Delta \bar{\psi}+|\psi|^{2 \sigma} \bar{\psi}\right) d W d x \\
& +\frac{1}{2} \sum_{\ell \in \mathbb{N}^{N}} \int_{0}^{\tau} \int_{\mathbb{R}^{n}}\left|\nabla \Phi e_{\ell}\right|^{2} d x d s \\
& -\frac{1}{2} \sum_{\ell \in \mathbb{N}} \int_{0}^{\tau} \int_{\mathbb{R}^{n}}\left[|\psi|^{2 \sigma}\left|\Phi e_{\ell}\right|^{2}+2 \sigma|\psi|^{2 \sigma-2}\left(\operatorname{Re}\left(\bar{\psi} \Phi e_{\ell}\right)\right)^{2}\right] d x d s
\end{aligned}
$$

where $\psi(\cdot)$ is the solution of (1.4) given by Theorem 2.1 with $\psi(0)=\psi_{0}$.

Using the preceding proposition, the following global existence result was proved in [4] in the subcritical case.

Theorem 2.2. If in addition to the assumptions of Theorem 2.1, $\sigma<2 / n$, then for any $\mathcal{F}_{0}$-measurable $\psi_{0}$, the solution of $(1.4)$ with $\psi(0)=\psi_{0}$ given by Theorem 2.1 is global, i.e. $\tau^{*}\left(\psi_{0}\right)=+\infty$ a.s. 
Note that the result of Theorem 2.1 is still true with $L^{2}$ solutions instead of $H^{1}$ solutions, if $0 \leq \sigma \leq 2 / n$ and if $\Phi$ is only Hilbert-Schmidt in $L^{2}\left(\mathbb{R}^{n}\right)$. In this case, the solutions are global, due to the estimate $(2.1)$ on the $L^{2}$ norm.

2.2. Blow-up for some initial data. The blow-up result for a restricted class of initial data, which is proved exactly as in [5], is based on Proposition 2.1, together with another identity, which we call the "stochastic variance identity". This identity is proved in [5] in the case of a complex valued noise - the real noise case is proved exactly in the same way - and requires slightly more regularity on $\Phi$ and $\psi_{0}$. In order to state precisely the assumptions we need, we introduce the space

$$
\Sigma=\left\{v \in H^{1}\left(\mathbb{R}^{n}\right), \int_{\mathbb{R}^{n}}|x|^{2}|v(x)|^{2} d x<\infty\right\} .
$$

Proposition 2.2. Let $\psi_{0}, \sigma$ and $\Phi$ be as in Theorem 2.1, and assume furthermore that $\Phi$ is Hilbert-Schmidt form $L^{2}\left(\mathbb{R}^{n}\right)$ into $\Sigma$ and that $\psi_{0}$ lies almost surely in $\Sigma$. Then for any stopping time $\tau$ such that $\tau<\tau^{*}\left(\psi_{0}\right)$ a.s. the solution $\psi$ of (1.4) with $\psi(0)=\psi_{0}$ belongs to $L^{\infty}(0, \tau ; \Sigma)$ a.s. and satisfies

$$
\begin{aligned}
V(\psi(\tau))= & V\left(\psi_{0}\right)+4 G\left(\psi_{0}\right) \tau+8 H\left(\psi_{0}\right) \tau^{2}+4 \frac{2-\sigma n}{\sigma+1} \int_{0}^{\tau}(\tau-s)|\psi|_{L^{2 \sigma+2}}^{2 \sigma+2} d s \\
& +c_{\Phi}^{\Sigma} \tau+\frac{4}{3} c_{\Phi}^{1} \tau^{3} \\
& -4 \sum_{\ell \in \mathbb{N}} \int_{0}^{\tau}(\tau-s)^{2} \int_{\mathbb{R}^{n}}|\psi|^{2 \sigma}\left|\Phi e_{\ell}\right|^{2}+2 \sigma|\psi|^{2 \sigma-2}\left(\operatorname{Re}\left(\bar{\psi} \Phi e_{\ell}\right)\right)^{2} d x d s \\
& +2 \operatorname{Im} \int_{\mathbb{R}^{n}} \int_{0}^{\tau}|x|^{2} \bar{\psi} d W d x \\
& -16 \operatorname{Im} \int_{\mathbb{R}^{n}} \int_{0}^{\tau} \int_{s^{0}}^{s_{2}} \int_{0}^{s_{1}}\left(\Delta \bar{\psi}+|\psi|^{2 \sigma} \bar{\psi}\right) d W(r) d s_{1} d s_{2} d x \\
& +4 R e \sum_{\ell \in \mathbb{N}} \int_{0}^{\tau_{0}} \int_{\mathbb{R}^{n}} \bar{\psi}\left(2 x \cdot \nabla \Phi e_{\ell}+n \Phi e_{\ell}\right) d x d \beta_{\ell} d s
\end{aligned}
$$

with

for $v \in \Sigma$, and with

$$
G(v)=\operatorname{Im} \int_{\mathbb{R}^{n}} v(x) x \cdot \nabla \bar{v}(x) d x
$$

$$
c_{\Phi}^{\Sigma}=\sum_{\ell \in \mathbb{N}} \int_{\mathbb{R}^{n}}|x|^{2}\left|\Phi e_{\ell}\right|^{2} d x \text { and } c_{\phi}^{1}=\sum_{\ell \in \mathbb{N}}\left|\nabla \phi e_{\ell}\right|_{L^{2}}^{2} .
$$

Note that the first four terms in this identity already occur in the deterministic identity, and that the other terms vanish in the absence of noise. The last three terms are stochastic integrals and are responsible for technical difficulties. In the particular case where $\tau=T$ is a deterministic time, the mean value of these last three terms vanishes. Let us denote $\mathbb{E}_{\Omega_{0}}(f)=\mathbb{E}\left(f \mathbb{1}_{\Omega_{0}}\right) / \mathbb{P}\left(\Omega_{0}\right)$ for $f \in L^{1}\left(\Omega_{0}\right)$ or $f$ measurable and nonnegative on $\Omega_{0}$, and $\Omega_{0}$ a $\mathcal{F}_{0}$ measurable set.

In the preceding case of a deterministic $\tau=T$, and if we assume moreover that

$$
\mathbb{E}_{\Omega_{0}}\left(\int_{0}^{T}|\nabla \psi(s)|_{L^{2}}^{2}+|\psi(s)|_{L^{2 \sigma+2}}^{2 \sigma+2} d s\right)
$$

is finite, then Proposition 2.1 implies that

$$
\mathbb{E}_{\Omega_{0}}(V(\psi(t))) \leq \mathbb{E}_{\Omega_{0}}\left(V\left(\psi_{0}\right)\right)+\left(4 \mathbb{E}_{\Omega_{0}}\left(\left|G\left(\psi_{0}\right)\right|\right)+c_{\Phi}^{\Sigma}\right) t+8 \mathbb{E}_{\Omega_{0}}\left(H\left(\psi_{0}\right)\right) t^{2}+\frac{4}{3} c_{\Phi}^{1} t^{3}
$$


for any $t \in[0, T]$. Now, it is possible to choose $\psi_{0}$ in such a way that the right hand side polynomial takes negative values for some $t \in[0, T]$, leading to a contradiction since the left hand side is nonnegative. More precisely, we have

Proposition 2.3. Assume that $2 / n \leq \sigma<2 /(n-2)$, and that $\Phi$ is Hilbert-Schmidt from $L^{2}\left(\mathbb{R}^{n}\right)$ into $\Sigma$. For each $\bar{T}>0, \bar{V}>0, \bar{G}>0, \bar{H}_{1}>0$, let $\bar{H}_{2}$ be such that

$$
\bar{V}+\left(4 \bar{G}+c_{\Phi}^{\Sigma}\right) \bar{T}+4\left(\bar{H}_{1}-\frac{1}{\sigma+1} \bar{H}_{2}\right) \bar{T}^{2}+\frac{4}{3} c_{\Phi}^{1} \bar{T}^{3}<0 ;
$$

then for each $\mathcal{F}_{0}$ measurable $\psi_{0}$ with values in $\Sigma$ and for any $\Omega_{0} \in \mathcal{F}_{0}$ with $\mathbb{P}\left(\Omega_{0}\right)>$ 0 such that

$$
\begin{aligned}
& \mathbb{E}_{\Omega_{0}}\left(\left|\nabla \psi_{0}\right|_{L^{2}}^{2}\right) \leq \bar{H}_{1}, \quad \mathbb{E}_{\Omega_{0}}\left(\left|G\left(\psi_{0}\right)\right|\right) \leq \bar{G} \\
& \mathbb{E}_{\Omega_{0}}\left(V\left(\psi_{0}\right)\right) \leq \bar{V} \quad \text { and } \quad \mathbb{E}_{\Omega_{0}}\left(\left|\psi_{0}\right|_{L^{2 \sigma+2}}^{2 \sigma+2}\right) \geq \bar{H}_{2},
\end{aligned}
$$

then either

$$
\mathbb{P}\left(\tau^{*}\left(\psi_{0}\right)<\bar{T}\right) \text { is positive }
$$

or

$$
\mathbb{E}_{\Omega_{0}}\left(\int_{0}^{\bar{T}}\left(|\nabla \psi(s)|_{L^{2}}^{2}+|\psi(s)|_{L^{2 \sigma+2}}^{4 \sigma+2}\right) d s\right)=+\infty,
$$

where $\psi$ is the solution of (1.4) with $\psi(0)=\psi_{0}$ given by Theorem 2.1.

The possibility that (2.7) occurs instead of (2.6) is due to the fact that we had to choose a deterministic time $T$ in the argument above in order to cancel the mean value of the stochastic terms in the stochastic variance identity. If instead we use a stopping time, then the expectation of those terms do not vanish. Under more restrictive assumptions on $\sigma$ and $\Phi$, however, they may be handled and lead to the following result.

Proposition 2.4. Assume that $2 / n<\sigma<\min \left(\frac{2}{3}, \frac{2}{n-2}\right)$, that $\Phi$ is Hilbert-Schmidt from $L^{2}\left(\mathbb{R}^{n}\right)$ into $\Sigma$, with moreover $\sum_{\ell \in \mathbb{N}}\left|\Phi e_{\ell}\right|_{L^{4 \sigma+2}}^{2}<\infty$, and bounded from $L^{2}\left(\mathbb{R}^{n}\right)$ into $H^{2}\left(\mathbb{R}^{n}\right) \cap L^{\infty}\left(\mathbb{R}^{n}\right)$. Let $\bar{T}, \bar{V}, \bar{G}, \bar{H}_{1}, \bar{H}_{2}, \Omega_{0}$ and $\psi_{0}$ be as in Proposition 2.2, with moreover, $\mathbb{E}_{\Omega_{0}}\left(M\left(\psi_{0}\right)^{1 /(1-\sigma)}\right)<\infty$; then

$$
\mathbb{P}\left(\tau^{*}\left(\psi_{0}\right)<\bar{T}\right)>0 .
$$

Note that the assumptions on the power $\sigma$ in Proposition 2.4 are compatible only when $n \geq 4$.

\section{The CONTROLABILITY PROBLEM AND BLOW-UP FOR ANY INITIAL DATA}

In this section, we prove the irreducibility of equation (1.4), or equivalently of the following equation :

$$
v(t)=S(t) \psi_{0}-i \int_{0}^{t} S(t-s)\left(|v+z|^{2 \sigma}(v+z)\right) d s
$$

where $z$ is given by (1.5), in the case where the noise is nondegenerate. (Note that if $z$ is given by (1.5) and $v$ satisfies (3.1), then $\psi=v+z$ is a solution of (1.4) with $\psi(0)=\psi_{0}$.) This is done by using the following controlability result, which was already proved in [5] in the case of a complex valued noise, together with some continuity property. The controlability result is more difficult to prove in the real 
valued case, because we have to control the NLS equation by using a real valued forcing term instead of a complex valued one, which means that we can act only on one of the two components of the solution. The result is also weaker in the sense that it requires more smoothness on the forcing term and on $\psi_{0}$. Of course, this will affect the main result in consequence (see Theorem 3.1).

We define some other spaces which are necessary to state the assumptions : for $k, \ell \in \mathbb{N}$ we define

$$
\Sigma^{k, \ell}=\left\{v \in H^{k+\ell}\left(\mathbb{R}^{n}\right),|x|^{\ell} v \in H^{k}\left(\mathbb{R}^{n}\right)\right\}
$$

endowed with the natural norm

$$
|v|_{\Sigma^{k, \ell}}^{2}=|v|_{H^{k+\ell}}^{2}+\left.\left.|| x\right|^{\ell} v\right|_{H^{k}} ^{2}
$$

and let $S_{n}$ be the following space (recall that $n$ is here the dimension of the space variable) :

$$
S_{1}=\Sigma, \quad S_{2}=\cup_{\alpha>0} \Sigma^{\alpha, 1}, \quad S_{n}=\Sigma^{1,2} \text { if } n \geq 3 .
$$

In order to understand how Proposition 3.1 below is related to the controlability of the NLS equation by a real valued forcing, one only needs to note that $\tilde{u}$ is a solution of

$$
\left\{\begin{array}{l}
i \frac{d \tilde{u}}{d t}-\left(\Delta \tilde{u}+|\tilde{u}|^{2 \sigma} \tilde{u}\right)=\frac{d f}{d t} \\
\tilde{u}(0)=\psi_{0}
\end{array}\right.
$$

if and only if $\tilde{u}=v+\tilde{z}$ with $\tilde{z}$ satisfying

$$
\left\{\begin{array}{l}
i \frac{d \tilde{z}}{d t}-\Delta \tilde{z}=\frac{d f}{d t} \\
\tilde{z}(0)=0
\end{array}\right.
$$

and $v$ satisfies equation (3.1) with $z$ replaced by $\tilde{z}$. In addition, $f(t)$ is real valued if and only if $i \tilde{z}(t)-\int_{0}^{t} \Delta \tilde{z}(s) d s$ is real valued.

Proposition 3.1. For any $T_{1}>0, \psi_{0} \in S_{n} \cap H^{s}, s>n / 2$, for any real valued function $b_{1}$ in $S_{n}$, there exists a $\tilde{z}$ in $C\left(\left[0, T_{1}\right] ; S_{n}\right)$ such that $\tilde{z}(0)=0$, iz $(t)-$ $\int_{0}^{t} \Delta \tilde{z}(s) d s$ is real valued for any $t \in\left[0, T_{1}\right]$ and such that the solution $v\left(\tilde{z}, \psi_{0}, \cdot\right)$ of (3.1) exists on $\left[0, T_{1}\right]$, with

$$
\operatorname{Im}\left(\tilde{z}\left(T_{1}\right)+v\left(\tilde{z}, \psi_{0}, T_{1}\right)\right)=b_{1} .
$$

Moreover, for any $\delta>0, \tilde{z}$ may be chosen so that

$$
\left|\operatorname{Re}\left(\tilde{z}\left(T_{1}\right)+v\left(\tilde{z}, \psi_{0}, T_{1}\right)\right)-\operatorname{Re}\left(\psi_{0}\right)\right|_{S_{n}} \leq \delta .
$$

Remark 3.1. This result is weaker than what we proved in [5] for a complex valued noise in two ways. First, we are not able to choose $\tilde{z}(\cdot)$ such that $\tilde{z}\left(T_{1}\right)+$ $v\left(\tilde{z}, \psi_{0}, T_{1}\right)=u_{1}$ for some fixed $u_{1}$; only the imaginary part can be controled exactly. However this is not a problem for our purpose as will be made clear later. Also, here we have to assume that $\psi_{0}, b_{1}$ have extra smoothness assumptions if $n \geq 2$ : they are assumed to be in $S_{n} \cap H^{s}\left(\mathbb{R}^{n}\right)$. This is the reason why Theorem 3.1 below is restricted to initial data in $S_{n} \cap H^{s}\left(\mathbb{R}^{n}\right)$. 
Proof of Proposition 3.1. It is convenient in this proof to decompose the solution of the nonlinear Schrödinger equation into its real and imaginary parts. We first construct the forcing $f$ in (3.2) and then deduce $\tilde{z}$ solution of (3.3) by the formula

$$
\tilde{z}(t)=-i f(t)-\int_{0}^{t} S(t-s)(i \Delta) f(s) d s .
$$

We note that $\tilde{u}$ solves

$$
i \frac{d \tilde{u}}{d t}-\left(\Delta \tilde{u}+|\tilde{u}|^{2 \sigma} \tilde{u}\right)=\frac{d f}{d t}
$$

for a real valued $f$ if its real and imaginary parts $a$ and $b$ solve

$$
\frac{d a}{d t}-\Delta b-\left(a^{2}+b^{2}\right)^{\sigma} b=0,
$$

and

$$
-\frac{d b}{d t}-\Delta a-\left(a^{2}+b^{2}\right)^{\sigma} a=\frac{d f}{d t} .
$$

The idea is to construct $b$ explicitly such that $b(0)=\operatorname{Im}\left(\psi_{0}\right), b\left(T_{1}\right)=b_{1}$ and $b=0$ on a large interval in $\left(0, T_{1}\right)$. In that way $\frac{d a}{d t}=0$ in that interval and $a\left(T_{1}\right)$ is close to $a(0)$.

Take $k_{1} \in \mathbb{N}$ and denote by $U(t)$ the semigroup on $\Sigma$ associated to the linear equation

$$
\left\{\begin{array}{l}
\frac{d w}{d t}+(-\Delta)^{k_{1}} w+|x|^{2 k_{1}} w=0, \quad x \in \mathbb{R}^{n} \\
w(0)=\psi_{0} .
\end{array}\right.
$$

For $\varepsilon>0$ to be chosen, we set

$$
b(t)= \begin{cases}\frac{\varepsilon-t}{\varepsilon} U(t) \operatorname{Im}\left(\psi_{0}\right), & t \in[0, \varepsilon], \\ 0, & t \in\left[\varepsilon, T_{1}-\varepsilon\right] \\ \frac{t-T_{1}+\varepsilon}{\varepsilon} U\left(T_{1}-t\right) b_{1}, & t \in\left[T_{1}-\varepsilon_{1}, T_{1}\right] .\end{cases}
$$

Clearly, for $k_{1}$ large enough, $b$ is in $C\left(\left[0, T_{1}\right] ; S_{n}\right) \cap L^{1}\left(0, T_{1} ; H^{s}\left(\mathbb{R}^{n}\right)\right)$ and $\Delta b$ is in $L^{1}\left(0, T_{1} ; S_{n} \cap H^{s}\left(\mathbb{R}^{n}\right)\right)$. Then, for any $\delta>0$, there exists $\varepsilon>0$ depending on $\psi_{0}$ and $b_{1}$ such that (3.4) has a solution $a$ in $C\left([0, \varepsilon] ; S_{n} \cap H^{s}\left(\mathbb{R}^{n}\right)\right)$ such that

$$
a(0)=\operatorname{Re}\left(\psi_{0}\right)
$$

and

$$
|a-a(0)|_{C\left([0, \varepsilon] ; S_{n} \cap H^{s}\right)} \leq \frac{\delta}{2} .
$$

This can be proved by a fixed point argument.

Similarly, there exists a solution $a$ of $(3.4)$ on $\left[T_{1}-\varepsilon, T_{1}\right]$ such that $a\left(T_{1}-\varepsilon\right)=$ $a(\varepsilon)$ and

$$
|a-a(\varepsilon)|_{C\left(\left[T_{1}-\varepsilon, T_{1}\right] ; S_{n} \cap H^{s}\right)} \leq \frac{\delta}{2} .
$$

Then, since $b=0$ on $\left[\varepsilon, T_{1}-\varepsilon\right]$, setting $a(t)=a(\varepsilon)$ on $\left[\varepsilon, T_{1}-\varepsilon\right]$ we obtain a solution of $(3.4)$ on $\left[0, T_{1}\right]$ such that

$$
\left|a-\operatorname{Re}\left(\psi_{0}\right)\right|_{C\left(\left[0, T_{1}\right] ; S_{n} \cap H^{s}\right)} \leq \delta .
$$


We now define

$$
f(t)=-b(t)+b(0)-\int_{0}^{t} \Delta a+\left(a^{2}+b^{2}\right)^{\sigma} a d s
$$

and

$$
\tilde{z}(t)=-f(t)-i \int_{0}^{t} S(t-s) \Delta f(s) d s
$$

so that $v=a+i b-\tilde{z}$ solves

$$
i \frac{d v}{d t}-\Delta v=|\tilde{u}|^{2 \sigma} \tilde{u}
$$

with $\tilde{u}=a+i b$, and for any $t \in\left[0, T_{1}\right], i \tilde{z}(t)-\int_{0}^{t} \Delta \tilde{z}(s) d s=f(t)$ is real valued.

We can choose $k_{1}$ in the definition of $U(\cdot)$ sufficiently large to ensure that $|b|^{2 \sigma+1} \in L^{1}\left(0, T_{1} ; S_{n}\right)$. Moreover, since $S_{n} \cap H^{s}$ is an algebra, we also have $|a|^{2 \sigma+1} \in L^{1}\left(0, T_{1} ; S_{n}\right)$. It follows that $|\tilde{u}|^{2 \sigma} \tilde{u} \in L^{1}\left(0, T_{1} ; S_{n}\right)$ and since $(S(t))_{t \in \mathbb{R}}$ is strongly continuous in $S_{n}$ and $v(0) \in S_{n}, v \in C\left(\left[0, T_{1}\right] ; S_{n}\right)$. Since we know that $\tilde{u}$ also belongs to this space, we have proved :

$$
\tilde{z} \in C\left(\left[0, T_{1}\right] ; S_{n}\right),
$$

and this ends the proof of Proposition 3.1.

The following corollary of Proposition 3.1 shows that it is possible to reach a state which leads to blow-up by controlling only the imaginary part of the solution.

Corollary 3.1. For any $\bar{T}_{1}, \bar{T}>0, \psi_{0} \in S_{n} \cap H^{s}\left(\mathbb{R}^{n}\right)$ for some $s>n / 2$, there exist $\bar{V}>0, \bar{G}>0, \bar{H}_{1}>0, \bar{H}_{2}>0$ satisfying (2.4) and there exists $\tilde{z} \in C\left(\left[0, T_{1}\right] ; S_{n}\right)$ such that $\tilde{z}(0)=0, i \tilde{z}(t)-\int_{0}^{t} \Delta \tilde{z}(s) d s$ is real valued for any $t \in\left[0, T_{1}\right], v\left(\tilde{z}, \psi_{0}, \cdot\right)$ exists on $\left[0, T_{1}\right]$ and $\tilde{u}_{1}=\tilde{z}\left(T_{1}\right)+v\left(\tilde{z}, \psi_{0}, T_{1}\right)$ verifies

$$
\left|\nabla \tilde{u}_{1}\right|_{L^{2}}^{2} \leq \frac{1}{2} \bar{H}_{1}, \quad\left|G\left(\tilde{u}_{1}\right)\right| \leq \frac{1}{2} \bar{G}, \quad V\left(\tilde{u}_{1}\right) \leq \frac{1}{2} \bar{V}, \quad \text { and } \quad\left|\tilde{u}_{1}\right|_{L^{2 \sigma+2}}^{2 \sigma+2} \geq 2 \bar{H}_{2} .
$$

Proof. Assume first that $\operatorname{Im}\left(\psi_{0}\right) \neq 0$; set $u_{\lambda}=\operatorname{Re}\left(\psi_{0}\right)+i \lambda \operatorname{Im}\left(\psi_{0}\right)$. Then the expression

$$
4 V\left(u_{\lambda}\right)+\left(16 G\left(u_{\lambda}\right)+c_{\Phi}^{\Sigma}\right) \bar{T}+4\left(4\left|\nabla u_{\lambda}\right|_{L^{2}}^{2}-\frac{1}{2(2 \sigma+2)}\left|u_{\lambda}\right|_{L^{2 \sigma+2}}^{2 \sigma+2}\right) \bar{T}^{2}+\frac{4}{3} c_{\Phi}^{1} \bar{T}^{3}
$$

is negative for $\lambda$ large enough, say larger than $\lambda_{0}$. Then, by Proposition 3.1 with $b_{1}=\lambda_{0} \operatorname{Im}\left(\psi_{0}\right)$, given $\delta>0$, we can find $\tilde{z}$ with $\tilde{z}(0)=0, i \tilde{z}(t)-\int_{0}^{t} \Delta \tilde{z}(s) d s$ is real valued for any $t \in\left[0, T_{1}\right], v\left(\tilde{z}, \psi_{0}, \cdot\right)$ exists on $\left[0, T_{1}\right]$ and with moreover

$$
\operatorname{Im}\left(\tilde{z}\left(T_{1}\right)+v\left(\tilde{z}, \psi_{0}, T_{1}\right)=\lambda_{0} \operatorname{Im}\left(\psi_{0}\right)\right.
$$

and

$$
\left|\tilde{z}\left(T_{1}\right)+v\left(\tilde{z}, \psi_{0}, T_{1}\right)-u_{\lambda_{0}}\right|_{S_{n}} \leq \delta
$$

Now, the quantities $|\nabla u|_{L^{2}}^{2},|G(u)|, V(u),|u|_{L^{2 \sigma+2}}^{2 \sigma+2}$ depend continuously on $u \in S_{n}$, so that choosing $\delta$ small enough, and setting $\tilde{u}(t)=\tilde{z}(t)+v\left(\tilde{z}, \psi_{0}, t\right)$, we have

$$
\left|\nabla \tilde{u}\left(T_{1}\right)\right|_{L^{2}}^{2} \leq 2\left|\nabla u_{\lambda_{0}}\right|_{L^{2}}^{2}, \quad\left|G\left(\tilde{u}\left(T_{1}\right)\right)\right| \leq 2\left|G\left(u_{\lambda_{0}}\right)\right|, \quad V\left(\tilde{u}\left(T_{1}\right)\right) \leq 2 V\left(u_{\lambda_{0}}\right)
$$
and

$$
\left|u\left(T_{1}\right)\right|_{L^{2 \sigma+2}}^{2 \sigma+2} \geq \frac{1}{2}\left|u_{\lambda_{0}}\right|_{L^{2 \sigma+2}}^{2 \sigma+2} .
$$

Hence we obtain the result with $\bar{H}_{1}=4\left|\nabla u_{\lambda_{0}}\right|_{L^{2}}^{2}, \bar{G}=4\left|G\left(u_{\lambda_{0}}\right)\right|, \bar{V}=4 V\left(u_{\lambda_{0}}\right)$ and $\bar{H}_{2}=\frac{1}{4}\left|u_{\lambda_{0}}\right|_{L^{2 \sigma+2}}^{2 \sigma+2}$. 
Assume now that $\operatorname{Im}\left(\psi_{0}\right)=0$, and let us consider the solution $\tilde{u}$ of $(3.2)$ with $f=0$; it is not difficult to see that the imaginary part of $\tilde{u}$ cannot be identically zero on a whole time interval $\left[0, t_{0}\right]$ with $t_{0}>0$; indeed, writting $\tilde{u}$ as $a+i b$ with $a$ and $b$ satisfying respectively (3.4) and (3.5), this would imply that $a$ is a stationary solution of the deterministic NLS equation on $\left[0, t_{0}\right]$, but it is well known that there is no such solution in $H^{1}\left(\mathbb{R}^{n}\right)$. Hence, for some positive $t_{0}$, one has $\operatorname{Im}\left(\tilde{u}\left(t_{0}\right)\right) \neq 0$, and we can reproduce the preceding argument by starting at time $t_{0}$ from $\tilde{u}\left(t_{0}\right)$ (which corresponds to taking $\tilde{z} \equiv 0$ on $\left[0, t_{0}\right]$ ).

We have now all the tools in hand to state and prove the main result.

Theorem 3.1. Assume that $2 / n \leq \sigma<\frac{2}{n-2}$ if $n \geq 3$, or $2 / n \leq \sigma$ if $n=1$ or 2 , that $\Phi$ is Hilbert-Schmidt from $L^{2}\left(\mathbb{R}^{n}\right)$ into $S_{n}$ and that the null space of $\Phi^{*}$ is reduced to $\{0\}$. Then for any $\psi_{0} \in S_{n} \cap H^{s}$ for some $s>n / 2$, and for any $t>0$ the solution $\psi(t)$ starting from $\psi_{0}$ and given by Theorem 2.1 satisfies either

$$
\mathbb{P}\left(\tau^{*}\left(\psi_{0}\right)<\bar{T}\right)>0
$$

or

$$
\mathbb{E}\left(\int_{0}^{\bar{T}}\left(|\nabla \psi(s)|_{L^{2}}^{2}+|\psi(s)|_{L^{2 \sigma+2}}^{4 \sigma+2}\right) d s\right)=+\infty
$$

If furthermore $\Phi$ satisfies the assumptions of Proposition 2.4, then $\psi$ blows up with a positive probability.

Proof of Theorem 3.1. The proof follows exactly the same lines as the proof of Theorem 2.1 in [5], once we have Corollary 3.1 in hand. We repeat shortly the arguments for the sake of completeness. Let $\bar{T}_{1}, \bar{T}>0$, and $\psi_{0} \in S_{n} \cap H^{s}\left(\mathbb{R}^{n}\right)$ with $s>n / 2$. Applying Corollary 3.1, we get $\bar{V}, \bar{G}, \bar{H}_{1}$, and $\bar{H}_{2}$ satisfying (2.4), and $\tilde{z} \in C\left(\left[0, T_{1}\right] ; S_{n}\right)$ such that if we set $\tilde{u}(t)=\tilde{z}(t)+v\left(\tilde{z}, \psi_{0}, t\right)$ then

$\left|\nabla \tilde{u}\left(T_{1}\right)\right|_{L^{2}}^{2} \leq \frac{1}{2} \bar{H}_{1}, \quad\left|G\left(\tilde{u}\left(T_{1}\right)\right)\right| \leq \frac{1}{2} \bar{G}, \quad V\left(\tilde{u}\left(T_{1}\right)\right) \leq \frac{1}{2} \bar{V}$, and $\left|\tilde{u}\left(T_{1}\right)\right|_{L^{2 \sigma+2}}^{2 \sigma+2} \geq 2 \bar{H}_{2}$.

Now, for $t \in\left[0, T_{1}\right]$, the mapping $z \mapsto v\left(\psi_{0}, z, t\right)$ is continuous on a neighbourhood of $\tilde{z}$ in $C\left(\left[0, T_{1}\right], \Sigma\right) \cap L^{r}\left(0, T_{1} ; W^{1,2 \sigma+2}\right) \cap L^{1}\left(0, T_{1} ; S_{n}\right)$ with values in $H^{1}\left(\mathbb{R}^{n}\right)$, and lower semi-continuous with values in $\Sigma$ (see Proposition 3.4 and 3.5 in [5]). Hence, there is a ball $B$ centered at $\tilde{z}$ in the preceding space, such that for any $z \in B$, $u=z+v\left(z, \psi_{0}, \cdot\right)$ exists on $\left[0, T_{1}\right]$ and satisfies

$$
\left|\nabla u\left(T_{1}\right)\right|_{L^{2}}^{2} \leq \bar{H}_{1}, \quad\left|G\left(u\left(T_{1}\right)\right)\right| \leq \bar{G}, \quad\left|V\left(u\left(T_{1}\right)\right)\right| \leq \bar{V}, \quad \text { and } \quad\left|u\left(T_{1}\right)\right|_{L^{2 \sigma+2}}^{2 \sigma+2} \geq \bar{H}_{2} .
$$

The solution of (1.4) with $\psi(0)=\psi_{0}$ is given by $\psi(t)=z(t)+v\left(z, \psi_{0}, t\right)$ with

$$
z(t)=\int_{0}^{t} S(t-s) d W(s)
$$

almost surely on $\left[0, \tau^{*}\left(\psi_{0}\right)\right)$. Since $\Phi$ is Hilbert-Schmidt from $L^{2}\left(\mathbb{R}^{n}\right)$ into $S_{n}, z$ is almost surely in $C\left(\left[0, T_{1}\right] ; S_{n}\right)$ (see [3], Theorem 6.10). Moreover, it is shown in [4] that $z$ is almost surely in $L^{r}\left(0, T ; W^{1,2 \sigma+2}\right)$. Since the null space of $\Phi^{*}$ is equal to $\{0\}, \Phi$ has dense range in $S^{n}$ and in $W^{1,2 \sigma+2}$, we deduce that $z$ is non-degenerate (note that $z$ is a Gaussian process with values in $S_{n}$ ) and $\mathbb{P}(z \in B)>0$; therefore the probability that $\tau^{*}\left(\psi_{0}\right) \geq T_{1}$ and $\psi\left(T_{1}\right)$ satisfies (3.6) is positive. We now set

$$
\Omega_{1}=\left\{\omega \in \Omega, \tau^{*}\left(u_{0}\right) \geq T_{1} \text { and } \psi\left(T_{1}\right) \text { satisfies (3.6) }\right\}
$$


A. DE BOUARD AND A. DEBUSSCHE

and note that $\psi\left(T_{1}\right), \Omega_{1}, \bar{T}, \bar{V}, \bar{G}, \bar{H}_{1}, \bar{H}_{2}$ satisfy the condition of Proposition 3.1 , or Proposition 3.2. The result follows.

\section{REFERENCES}

[1] O. Bang, P.L. Christiansen, F. If, K.O. Rasmussen, Y.B. Gaididei, Temperature effects in a nonlinear model of monolayer Scheibe aggregates, Phys. Rev. E 49, 4627-4636 (1994).

[2] O. Bang, P.L. Christiansen, F. If, K.O. Rasmussen, Y.B. Gaididei, White Noise in the Twodimensional Nonlinear Schrödinger Equation, Appl. Anal. 57, 3-15 (1995).

[3] G. Da Prato, J. Zabczyk, Stochastic equations in infinite dimensions, Encyclopedia of Mathematics and its Applications, Cambridge University Press (1992).

[4] A. de Bouard, A. Debussche, The stochastic nonlinear Schrödinger equation in $H^{1}$, to appear in Stoch. Anal. and Appl.

[5] A. de Bouard, A. Debussche, On the effect of a noise on the solutions of supercritical nonlinear Schrödinger equation, to appear in Probab. Theory Relat. Fields.

[6] A. Debussche, L. Di Menza Numerical simulation of focusing stochastic nonlinear Schrödinger equations. Preprint.

[7] G.E. Falkovich, I. Kolokolov, V. Lebedev, S.K. Turitsyn, Statistics of soliton-bearing systems with additive noise, Phys. Rev. E 63 (2001).

[8] R.T. Glassey, On the blowing-up of solutions to the Cauchy problem for the nonlinear Schrödinger equation, J. Math. Phys. 18 1794-1797 (1977).

[9] C.E. Kenig, G. Ponce, L. Vega, On the ill-posedness of some canonical dispersive equations, Duke Math. J. 106, 617-633 (2001).

[10] C. Sulem, P.L. Sulem, The Nonlinear Schrödinger Equation, Self-Focusing and Wave Collapse, Appl. Math. Sciences, Springer Verlag: New York, 1999.

[11] S.N. Vlasov, V.A. Petrishchev, V.I. Talanov, Average description of wave beams in linear and nonlinear media (The method of moments), Radiophys. Quantum Electron. 14 1062-1070 (1974).

[12] V.E. Zakharov, Collapse of Langmuir waves, Sov. Phys. JETP 35, 908-914 (1972).

CNRS et Université Paris-Sud,, UMR 8628, BÂt. 425, Université de Paris-Sud,, 91405 ORSAY CEDEX, FRANCE

E-mail address: Anne.deBouard@math.u-psud.fr

Ens de Cachan, Antenne de Bretagne, Campus de Ker Lann, Av. R. Schuman,, 35170 BRUZ, FRANCE

E-mail address: Arnaud.Debussche@bretagne.ens-cachan.fr 